\title{
INTERVENTION CARDIOLOGY
}




\title{
Coronary angiographic profile of Nepalese people A study of $\mathbf{4 5 0}$ cases in SGNHC
}

\author{
Dr S Regmi, Dr R Malla, Dr S Rajbhandari, Dr M.B.K.C, Dr D Sharma, Dr \\ Y Bhatt, Dr R Rajbhandari, Dr Y.R.Limbu, Dr B Koirala, Dr S Kansakar
}

\section{Background}

Coronary Artery disease (CAD) remains a major health problem. Stable angina, unstable angina and myocardial infarction are the major manifestations of CAD. This study is aimed to assess the coronary artery profile in these groups and its relation to the major conventional risk factors and the distribution of the disease in differant ethnic group of Napalase population.

\section{Method and Result}

A data of 450 patients who underwent coronary angiography in Shahid Gangalal National Heart Center from May-2002 to December-003 was analyzed retrospectively. Mean age of the patients were 55+11 in myocardial infarction group, 54+11 in stable angina group and 53+12 in unstable angina group, 328 (72.9\%) were male and $122(27.1 \%)$ were female. Regarding risk factors 180 (40\%) were smoker, DM in 1/1 (24.7\%) Dislipidemia $84(18.7 \%)$, Hypertension $246(54.7 \%)$ and family history of CAD was positive in 25 $(5.6 \%)$ cases.160 (35.6\%) had MI,102 (22.7\%) had UA,146 (32.4\%) had stable angina and $93(20,7 \%)$ underwent CAG for non coronary reason e.g for valvular surgery or surgery for peripheral arterial disease.

Among these 71 (15.8\%) had triple vessel disease, 93 (20.7\%) had double vessel disease, 155(34.4\%) had single vessel disease and 182 (40.4) had normal CAG finding. TVD was common in UA. Patients $(29.4 \%)$ than in MI group (15.6\%).

Left main disease was found on $4(0.01 \%)$ Ramus intermedius was present on $15(3.3 \%)$ 442 (98.22\%) were Right dominant, 6 (1.3\%) were Laft dominant and $2(0.4 \%)$ were codominant. 
Complication during procedure occurred in $10(0.02 \%)$ cases (excluding damping and ventricularization). Among thease $1(0.002 \%)$ had CVA, $3(0.007 \%)$ had acute chest pain, $3(0.007 \%)$ had hypotension and $3(0.007 \%)$ had VT. No allergic reaction occurred.

TVD was common in Brahmin 16.3\%, then Newar 10.9\% ethnic group, 